\title{
Visual space under free viewing conditions
}

\author{
MICHELLE J. A. DOUMEN, ASTRID M. L. KAPPERS, and JAN J. KOENDERINK \\ Helmholtz Instituut, Utrecht, The Netherlands
}

\begin{abstract}
Most research on visual space has been done under restricted viewing conditions and in reduced environments. In our experiments, observers performed an exocentric pointing task, a collinearity task, and a parallelity task in a entirely visible room. We varied the relative distances between the objects and the observer and the separation angle between the two objects. We were able to compare our data directly with data from experiments in an environment with less monocular depth information present. We expected that in a richer environment and under less restrictive viewing conditions, the settings would deviate less from the veridical settings. However, large systematic deviations from veridical settings were found for all three tasks. The structure of these deviations was task dependent, and the structure and the deviations themselves were comparable to those obtained under more restricted circumstances. Thus, the additional information was not used effectively by the observers.
\end{abstract}

Humans are capable of interacting adequately with their surroundings on the basis of visual input. People can estimate the positions of objects well enough to interact with them effectively, but sometimes they make large errors in estimating the positions of objects. For example, when someone standing next to you points to a person who is surrounded by other people, it may be hard to locate the person whom he or she is actually referring to.

People tend to make significant and systematic errors when estimating the positions of objects in a visual scene. It is as if "visual space"- that is, how we visually perceive the world around us - is deformed with respect to physical space - the physical layout of the world. Over the years, many researchers have been interested in quantifying this deformation and in giving it a geometric description. Early experiments on visual space were generally done in dark rooms, where observers viewed stimuli binocularly while their head position was fixed. Such a setup effectively removes monocular distance cues, except for accommodation. Thus, in these experiments the focus was on binocular depth cues. Helmholtz (1962), for example, measured apparent frontoparallel planes. Vertical threads that hung in a physically frontoparallel plane were not judged by observers to be in one plane. Therefore, Helmholtz concluded that the apparent frontoparallel plane is not the same as the physical plane but is instead curved. Other early research was carried out with luminous points as stimuli; observers had to do visual tasks that involved rearranging the points. The experiments of Blumenfeld and Hillebrand, who let observers make visual alleys based on parallelity

The investigations reported here were supported by the Research Council for Earth and Life Sciences (ALW), with financial aid from the Netherlands Organization for Scientific Research (NWO). Correspondence relating to this article may be sent to M. J. A. Doumen, Helmholtz Instituut, Princetonplein 5, $3584 \mathrm{CC}$ Utrecht, The Netherlands (e-mail: m.j.a.doumen@phys.uu.nl). or equidistance, inspired Luneburg to formulate a model for visual space (Luneburg, 1950). For these kinds of tasks under these conditions, he suggested that visual space has a Riemannian geometry of constant hyperbolic curvature. Both Zajaczkowska (1956) and Blank (1961) confirmed this notion. However, Indow and Watanabe $(1984,1988)$ found that the metric of visual space varies over different planes in the visual world. They found a Euclidean metric for the frontoparallel plane (Indow \& Watanabe, 1984, 1988), and Indow (1991) found a curved Riemannian metric for the horizontal plane at eye height. These findings suggest that visual space is anisotropic, which contradicts Luneburg's assumption of isotropy. Because all of the experiments above were conducted in dark rooms, most monocular depth cues were not available to the observers. Thus, if we want to generalize this knowledge to everyday vision, we should extend the research with experiments done under normal lighting conditions.

Some researchers have concentrated on experiments in large open fields in normal daylight. In these open-field experiments, distances between objects and the observer are larger than in the laboratory. Thus, different kinds of information (mainly monocular) become important when observers do tasks involving the estimation of depth in a scene. For example, at distances of more than $4 \mathrm{~m}$, binocular depth cues play a less important role. Testing in daylight, in contrast with testing in the dark, provides depth information from pictorial cues such as linear perspective and texture. Gilinsky (1951), for example, did research aimed at obtaining insight into the relationship between the perceived distance and the perceived size of objects at distances of up to $22 \mathrm{~m}$ ( 70 feet). She developed a law to describe the compression of visual space perception found in her experiments. She described perceived distance $(P)$ with the following formula:

$$
P=\frac{c r}{c+r}
$$


where $r$ is the physical distance and $c$ is a constant that represents the distance at which an observer perceives objects that are an infinite distance away. In near space, perceived distance is approximately equal to physical distance, but as physical distance increases, perceived distance increases less and less, until it reaches asymptote at distance $c$.

Some scientists have concluded that there is no single geometry that can describe visual space under all conditions. For example, Battro, di Pierro Netto, and Rozestraten (1976) and Koenderink, van Doorn, and Lappin (2000) found that the curvature of visual space changes from elliptic to hyperbolic as the distance from the observer increases. In addition, Koenderink, van Doorn, Kappers, and Lappin (2002) concluded that the structure of visual space varies over different tasks. Thus, studies that confirm theories based on different geometries are not necessarily contradictory, merely complementary. According to Wagner (1985), visual space has an affine-transformed Euclidean geometry under full-cue conditions with free head movement but restricted body movement. The metric approaches Euclidean when perceptual information increases both quantitatively and qualitatively (Wagner, 1985).

Recently, Cuijpers, Kappers, and Koenderink (2000a, 2000b, 2001, 2002) did indoor experiments in a room where most pictorial depth cues were eliminated from the visual field by means of wrinkled plastic that prevented observers from seeing the walls. The floor and ceiling of the room were not visible because the observer was seated in a cabin that restricted the vertical field of view. The head of the observer was fixed using a chinrest. In the tasks in these experiments, the observer had to point rods toward a target (exocentric pointing task) or toward each other (collinearity task) or had to place a rod parallel to another rod (parallelity task). The angular deviations from veridical settings were then measured. The pattern of these deviations was found to depend on the task, leading Cuijpers et al. (2002) to claim that there is no such thing as an invariant visual space, because the form of visual space is task dependent.

Cuijpers et al.'s experiments (2000a, 2000b, 2001, 2002) were done with artificial light and concerned distances less than $4.5 \mathrm{~m}$, with most pictorial depth information eliminated from the scene. In this way, the information present came mainly from physiological depth cues. In everyday vision, however, pictorial depth information is available to an observer. Therefore, to be able to say something about everyday vision, one needs to look at how visual space is deformed when contextual information is present. Normally, people do not look at luminous points in a totally dark environment but instead look at objects that are surrounded by other objects that can give a great deal of information about the relative positions of the objects. This is evident from the fact that one obtains spatial impressions from flat photographs in which physiological cues are lacking or are inconsistent with monocular cues. According to Gibson (1950), visual space is dependent on what fills it; thus, in studying visual space one should also look at contextual information. Another example that stresses the amount of information that can be provided by monocular depth cues is the perception of amblyopes, people who are unable to use binocular depth information but nevertheless have no trouble perceiving depth in a normal environment. In fact, very often such observers only discover their deficiency when they are subjected to stereo tests.

Since monocular information provides rich information about depth in a scene, it seems logical to examine the perceived spatial relations between objects in an environment that provides both monocular and binocular depth cues. The purpose of the present research is to increase current understanding of the structure of visual space as it occurs when both types of depth cues are present. To do this, we studied the structure of visual space in an illuminated room under free viewing conditions - that is, without any restrictions on head movements or the size of the visual field during viewing. We tested whether visual space is systematically different from physical space under these conditions in a room where monocular depth information is available. Our hypothesis is that in a richer environment the settings for similar tasks will deviate less from veridical settings than they typically would in more restrictive environments. We expected this result because more pictorial depth cues are present, and thereby one would expect more precise estimation of the positions of objects (Wagner, 1985). Another issue in our research is whether the differences that Cuijpers et al. found between different tasks are also present with our setup.

The research was done in a room in our laboratory. The observers were seated, could rotate their head and upper body if they liked, and had an unobstructed view of the floor, ceiling, and walls of the experimental room. We used three different tasks: One was an exocentric pointing task, in which the observer had to direct a pointer toward a target. The second was a parallelity task, in which a rod had to be put parallel to another rod. The third was a collinearity task, in which two rods had to be placed in one line. We manipulated two different parameters for all three of the tasks - the relative distance, or the ratio of the distances between the two objects and the observer, and the separation angle, or the visual angle between the objects. For the parallelity task, we also had a third parameter-namely, the orientation of the reference rod. Relative distance and separation angle were chosen as parameters because, together, they can quite naturally give an indication of the positions of the objects with respect to the observer, and also because they were the major parameters in the experiments of Cuijpers et al. (2000a, 2000b, 2002). Since we want to test whether a room full of depth information will change the structure of visual space, it is important to be able to use Cuijpers et al.'s data as a baseline for our measurements.

\section{GENERAL METHOD}

\section{Observers}

All three tasks described in this article involved the same 4 observers (A.W., B.L., J.P., and T.L.). The observers were undergradu- 
ates and were paid for their efforts. All had normal or corrected-tonormal sight and were tested for binocular vision. All observers also had stereo vision with good acuity. The observers had no knowledge of the goal of the experiment and received no feedback regarding their performance. They were tested individually.

\section{Experimental Setup}

The experiment was set up in an empty room measuring $6 \times 6 \times$ $3 \mathrm{~m}$. On the left-hand wall, blinded windows were visible. Under the windows were central heating radiators. Opposite the observer was an empty wall, and on the observer's right was a wall with two doors. On every wall, electric sockets were visible near the floor of the room. The ceiling was partly covered with oblong fluorescent lights and air-conditioning equipment. The room was illuminated by the artificial lights. On the floor, points were marked for the positioning of the objects. These points were marked at three different distances from the observer $(1.5,2.6$, and $4.5 \mathrm{~m})$ at three different angular separations $\left(20^{\circ}, 40^{\circ}\right.$, and $\left.60^{\circ}\right)$ and were symmetrical around a line bisecting the room (see Figure 1). The objects used in the tasks consisted of yellow disks perpendicular to green rods. The rods were $25 \mathrm{~cm}$ long and $1.0 \mathrm{~cm}$ thick and were sharp at each end. The disks had a diameter of $8.2 \mathrm{~cm}$ and a thickness of $1.0 \mathrm{~cm}$. The rods were placed at eye height and could be rotated around the vertical axis. The rods that were used as objects are depicted in Figure 2. The observer used a remote control to rotate the rods. The feet of the objects were square shaped and contained a protractor from which the experimenter could read the pointing direction. A screen in front of the foot of an object prevented the observer from seeing the protractor and the square, which were aligned with the walls. The observer's chair could be adjusted so that the objects were at eye height. No chinrest was used, and head movements were permitted.

\section{Procedure}

For every trial, two objects were placed on the marks in the room. We used three different separation angles $\left(20^{\circ}, 40^{\circ}\right.$, and $\left.60^{\circ}\right)$, and the objects were at three different distances from the observer $(1.5,2.6$, and $4.5 \mathrm{~m}$ ). For every combination of positions on the floor, one object was always on the left of the observer and the other was always on the right. This setup allowed a total of 27 possible combinations of positions on the floor ( 3 separation angles $\times 3$ distances to one object $\times 3$ distances to the second object).

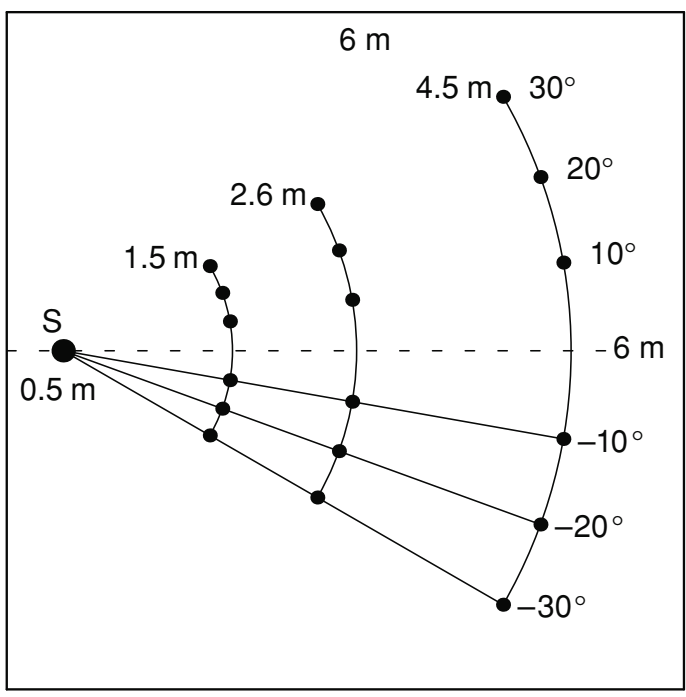

Figure 1. Schematic view of the experimental room. The small black dots indicate the positions of the objects in the room. The larger black dot represents the position of the observer (S).

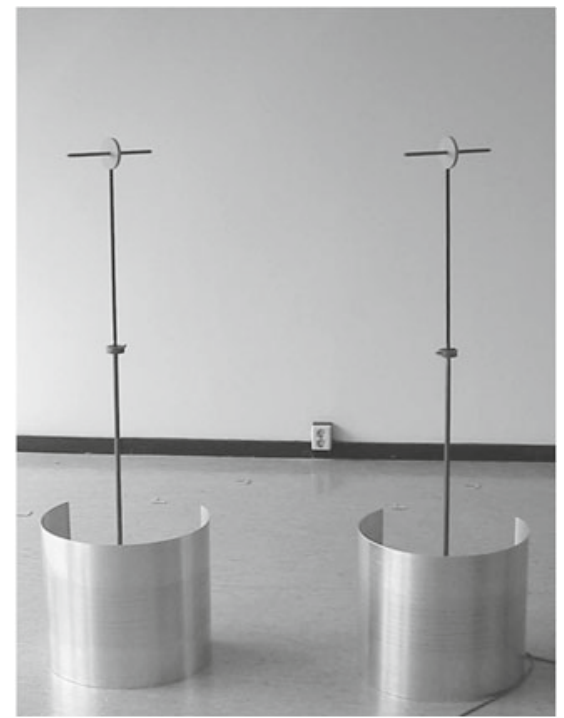

Figure 2. A picture of the two rods that were used in the parallelity task and the collinearity task.

For the analysis, we used positive and negative separation angles. Positive separation angles $\left(20^{\circ}, 40^{\circ}\right.$, and $\left.60^{\circ}\right)$ were used for the trials in which one object, the reference object, was positioned to the left of the observer and the other, the test object, was positioned to the right. Negative separation angles $\left(-20^{\circ},-40^{\circ}\right.$, and $\left.-60^{\circ}\right)$ were used when the reference object was to the right of the observer and the test object was to the left.

The observers were allowed to move their head but were told to stay seated. Between trials, the observers were asked to close their eyes so they could not see the movements of the experimenter and the objects while the experimenter read the pointing direction of the test rod and changed the setup for the next trial.

All observers were tested with all three tasks, and each task was completely finished before starting the next one. The order of the experiments was partially counterbalanced so that every observer had a unique order of experiments. The experiments were all conducted in sessions of approximately $1 \mathrm{~h}$. Mostly, the observers were tested for $1 \mathrm{~h}$ each day, but sometimes we had two sessions in a day, with a break of at least $30 \mathrm{~min}$ between the sessions.

\section{EXPERIMENT 1 Exocentric Pointing Task}

\section{Method}

The exocentric pointing task involved the use of a pointer and a target. The target was an orange sphere with a diameter of $6.5 \mathrm{~cm}$ and was positioned at the same height as the pointer, at eye height for the observer. For this task, an object like the ones described above was used as a pointer, but it had only one sharp conical end. The task was to rotate the pointer in such a way that it pointed toward the center of the target. Each position on the floor was used as both reference position (position of the target) and test position (position of the pointer), and we repeated every combination three times. Thus, the total number of trials needed for this task was $162(27 \times$ $2 \times 3$ ). It took each observer about $3 \mathrm{~h}$ to complete the 162 trials.

\section{Results}

In the exocentric pointing task, qualitatively similar systematic deviations were found for all observers, although the magnitude of the deviations was observer dependent. 
Figure 3 depicts an example of the settings for Observer A.W., with a separation angle of $60^{\circ}$ and a distance of $2.6 \mathrm{~m}$ to the target. The layout of the floor is presented in this figure, together with the three combinations of the positions of target and pointer that were possible with a fixed target position. The dotted lines represent the veridical pointing directions, and the small thick lines represent the means of three settings by the observer. The numbers indicate the deviation from the veridical direction, in degrees. It is important to notice that the sign changes when the relative distance from the observer switches from larger than 1.0 to smaller than 1.0.

The influence of two parameters, separation angle and relative distance, was analyzed. Figure 4 shows graphs for Observer A.W. in which the deviation is plotted against the separation angle. A line is fitted through the data points in the figure using a least squares method. The five graphs represent the five relative distances that were used. For relative distances of 0.3 and 0.6 , the slopes are positive, and for relative distances of 1.7 and 3.0, the slopes are negative. For a relative distance of 1.0, the fit approaches a horizontal line. Table 1 gives the slopes for each observer and each relative distance. An asterisk indicates slopes that deviate significantly from zero at a confidence level of .95. The same pattern was found for 3 of the observers; that is, the slope deviated significantly from zero when the two rods were at different distances from the observer. The deviations for Observer T.L. were very small, and therefore the slope values were smaller. The results for

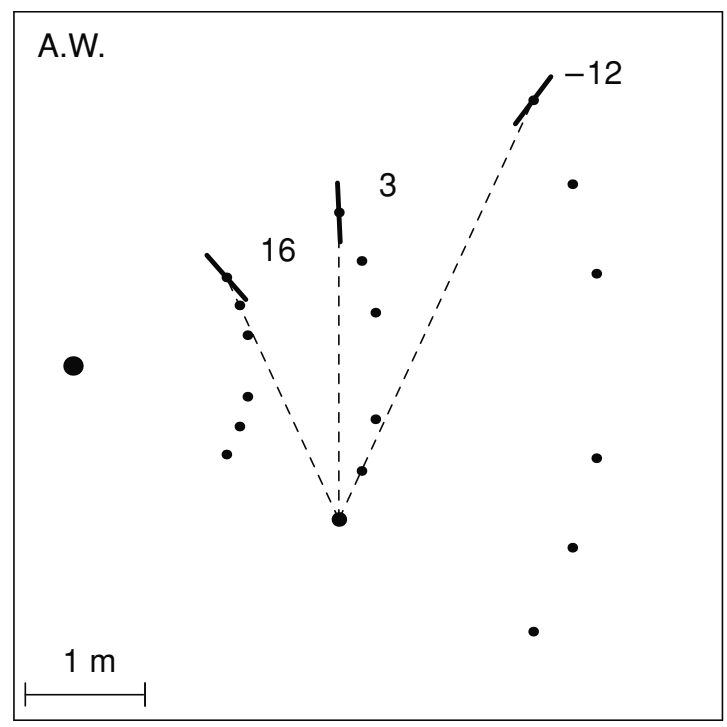

Figure 3. Schematic view of the experimental room with settings for the exocentric pointing task. The dashed lines represent veridical settings for the exocentric pointing task for conditions in which the separation angle was $-60^{\circ}$. The distance from the reference stimulus to the observer was $2.6 \mathrm{~m}$. The solid lines represent the means of the settings for three trials for Observer A.W. They are shown for three different distances between the observer and the pointer. The numbers indicate the mean deviations from the veridical settings, in degrees.
Observer B.L. also showed two slopes that did not deviate significantly from zero. However, in general the observed deviations could be approximated very well as a linear function of the separation angle.

Figure 5 is a graph in which the slopes of the lines in Figure 4 are plotted against the relative distance on a logarithmic scale. A large slope value (positive or negative) means that deviations were very large for the larger separation angles, and a small slope value indicates small deviations at the larger separation angles. The slopes in Figure 5 thus give indirect information about the size of the deviations at one relative distance. The data for the 4 observers are plotted on separate lines. As can be seen in the Figure 5, the lines have the same shape, which indicates that the observers showed comparable behavior. We will address three points: First, an overshoot was present when the relative distance was smaller than 1.0 (i.e., when the pointer was closer to the observer than was the target). This means that the observers directed the pointer toward a point farther away in depth than the target actually was. In contrast, an undershoot was present when the relative distance was larger than 1.0 (i.e., the pointer was farther away from the observer than was the target). This means that the observer directed the pointer toward a point closer to himself than the target actually was. Second, the size of the slopes approached zero for a relative distance of 1.0; when the target and the pointer were at the same distance from the observer, the settings were almost veridical. Third, the slope values were larger for relative distances of 0.6 and 1.7 than for relative distances of 0.3 and 3.0.

\section{Discussion}

The deviations increased linearly with the separation angle. This means that when the two objects were farther apart - that is, when the visual angle was large - the deviations were larger. An effect of relative distance was also found: There was an overshoot when the target was farther away from the observer than was the pointer and an undershoot when the target was closer. At relative distances of 0.6 and 1.7, the deviations were larger than those for relative distances of 0.3 and 3.0. This result could be due to the fact that when the relative distance approaches zero or infinity, the angle between the line connecting the observer with the more distant object and the line connecting the two objects approaches zero. In these cases, the task resembles an egocentric pointing task. Thus, the closer the relative distance approaches to zero or infinity, the smaller the deviations are likely to be.

When the two objects were at the same distance from the observer (a relative distance of 1.0), the settings of 2 of the observers (A.W. and T.L.) were close to veridical. For the other 2 observers, a small overshoot was present at this relative distance.

Comparing these results with the data of Cuijpers et al. (2000a), we see that the same pattern of results was found for relative distance, but Cuijpers et al. (2000a) did not find any effect of separation angle. However, they looked only at the separation angle for combinations of positions 


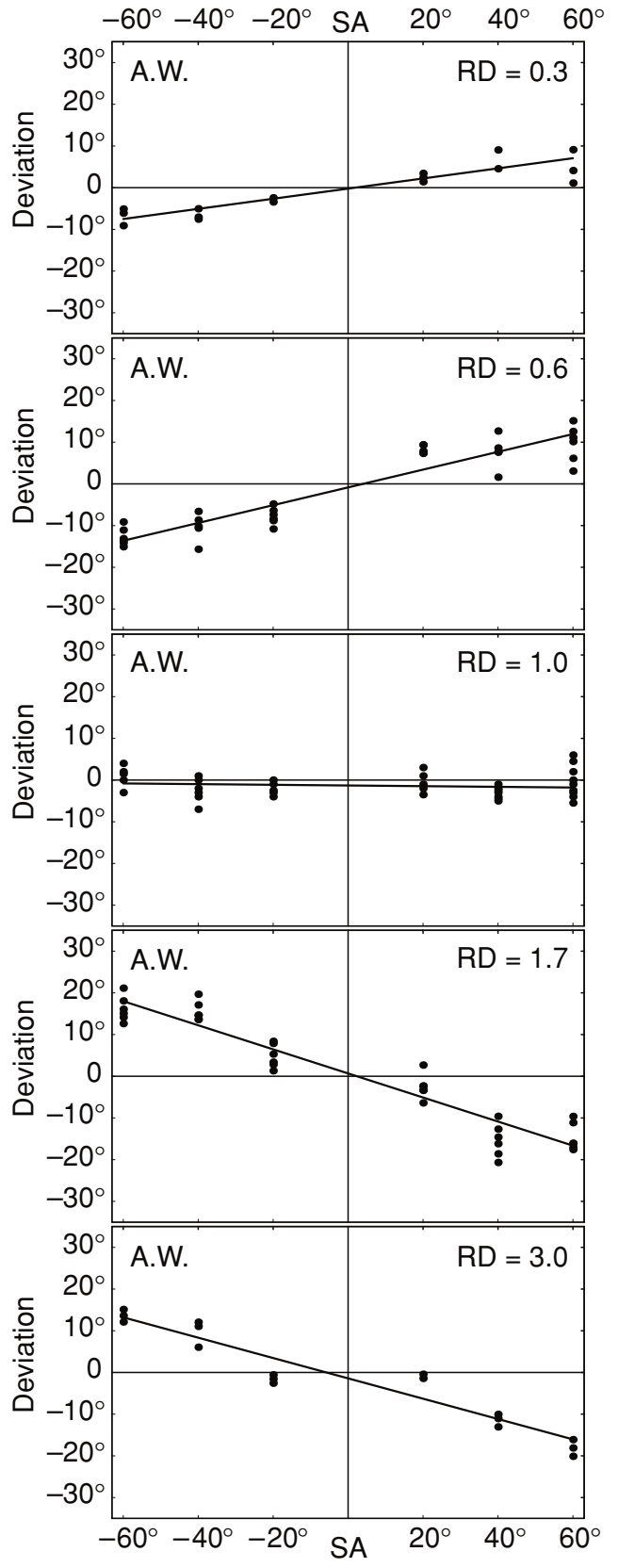

Figure 4. In each graph, the deviations from veridical settings for the exocentric pointing task are shown as a function of separation angle (SA) for each relative distance (RD). These are the data for Observer A.W.A line is fitted through each set of data points.

with a relative distance of 1.0, and for these trials we also found only minor deviations.

\section{EXPERIMENT 2 Parallelity Task}

\section{Method}

For the parallelity task, two rods were used, as described in the General Method section. One of the rods, the reference rod, was placed at a certain orientation by the experimenter. The task for the observer was to rotate the other, test, rod so that the two rods were parallel. To clarify the word parallel, we gave the observers an example of two parallel lines on paper. The reference rod could be either on the left or the right side of the room. Thus, as in the pointing experiment, the number of combinations of positions was doubled. Furthermore, four different orientations of the reference $\operatorname{rod}\left(22^{\circ}, 67^{\circ}, 112^{\circ}\right.$, and $\left.157^{\circ}\right)$ were used for every combination of the reference and test positions. We chose these orientations so we could compare four oblique orientations with an even amount of rotation between them. We repeated all of the measurements three times, resulting in 648 trials for each observer $(27 \times 2 \times 4 \times 3)$. Usually 54 trials were performed per session. Each session lasted an hour, so $12 \mathrm{~h}$ were needed per observer.

\section{Results}

An example of the settings of Observer B.L. with one distance to the reference rod and one orientation is given in Figure 6. The figure shows the layout of the floor of the experimental room. The point on the left represents the position of the observer, the other points the positions that were used for the rods. The figure gives both a graphical and a numerical view of the data. The lines and numbers (in degrees) represent the means of three trials for a reference orientation of $67^{\circ}$ and a distance of $4.5 \mathrm{~m}$ between observer and reference rod. For this reference distance, all possible combinations with the test distance are shown, as well as three different separation angles. The test rods on the outer line were tested with the reference rod on the outer line on the other side of the room (separation angle of $60^{\circ}$ ), and all rods on the middle and the inner lines were also tested together (separation angles of $40^{\circ}$ and $20^{\circ}$, respectively).

Systematic deviations from veridical settings were found for all of the observers. However, the size of the deviations was dependent on the observer; sizes varied from $0^{\circ}$ to $44^{\circ}$, and Observer A.W. produced the smallest deviations.

We will now look more closely at the three parameters that may influence the pattern of deviations found in this experiment. These parameters are the separation angle, the relative distance, and the reference orientation.

Figure 7 shows the graphs for Observer B.L. and a reference orientation of $22^{\circ}$; the deviations are plotted against the separation angle. Each graph represents the data for one relative distance, and each point in the graphs represents one trial. A line is fitted through these data points using a least squares method.

Table 1

Slopes of the Linear Fits Through the Data Points as a Function of Relative Distance for All Observers in the Exocentric Pointing Task

\begin{tabular}{lccccc}
\hline & \multicolumn{5}{c}{ Relative Distance } \\
\cline { 2 - 6 } Observer & 0.3 & 0.6 & 1.0 & 1.7 & 3.0 \\
\hline A.W. & $0.12^{*}$ & $0.21^{*}$ & -0.01 & $-0.29^{*}$ & $-0.24^{*}$ \\
B.L. & -0.02 & 0.04 & $-0.12^{*}$ & $-0.27^{*}$ & $-0.23^{*}$ \\
J.P. & $0.11^{*}$ & $0.18^{*}$ & $-0.08^{*}$ & $-0.17^{*}$ & $-0.07^{*}$ \\
T.L. & 0.01 & $0.12^{*}$ & 0 & $-0.07^{*}$ & -0.01 \\
\hline
\end{tabular}

Note-The data for A.W. are illustrated in Figure 4. ${ }^{*}$ The slope deviates significantly from $0(\alpha=.05)$. 


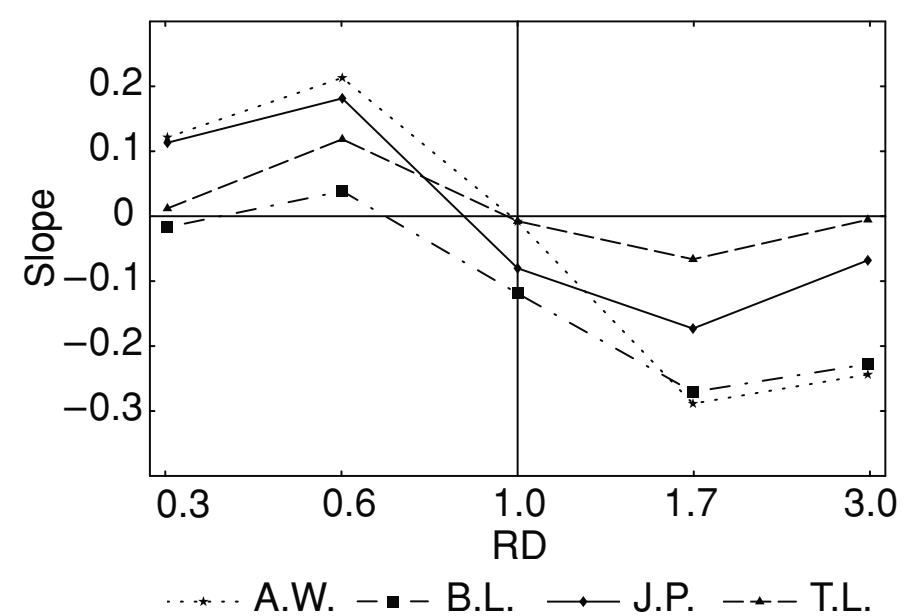

Figure 5. The slopes of the lines for deviations from veridical settings, as given in Figure 4 for Observer A.W., are plotted as a function of relative distance (RD) for all observers. The data for the different observers are given on separate lines.

The slopes of the fits that were plotted in Figure 7 are plotted in Figure 8 against the relative distance. Each graph contains the data for 1 observer. The data for the four reference orientations are given on separate lines, and the error bars indicate the confidence intervals for the slopes. The lines are nearly horizontal, and the points for each reference orientation are all within the range of the confidence intervals of the other points on the line, so the relative distance has no effect on the slope. The slope values represent the dependence of the deviations on the separation angle, giving an indication of the range of the deviations that were measured. The more the slope deviates from zero, the wider the range of deviations. Thus, our method yields a pattern that resembles the one produced by plotting the deviations directly against the relative distance. We not only considered the influence of relative distance, but also the effect of the absolute distance between the observer and the two rods. The absolute distance, however, had no effect on the size of the deviations.

In Figure 8, it can be seen that the lines representing the different reference orientations are very similar for 2 of the observers (B.L. and T.L.). The results for the other 2 observers (A.W. and J.P.) show an effect of the reference orientation. For reference orientations of $22^{\circ}$ and $157^{\circ}$, the effect of the separation angle was small (the slope values in Figure 8 are small), but for the other two orientations the effect of the separation angle was larger. For Observers A.W. and J.P., a significant difference was found between reference orientations of $22^{\circ} / 157^{\circ}$ and $67^{\circ} / 112^{\circ}$ (Student's $t$ test, $p<.0001$ for both observers), but this difference was not present for the other 2 observers ( $p=.12$ for T.L., .15 for B.L.).

The slopes of the linear fits of Figure 7 are shown in Table 2. The slope values are the means of all relative distances across two reference orientations $\left(22^{\circ}\right.$ and $157^{\circ}$, or $67^{\circ}$ and $112^{\circ}$ ). All slopes deviated from zero significantly at a confidence level of .95 .

\section{Discussion}

For the parallelity task, the deviations increased linearly with separation angle. No effect of distance was found. Thus, neither the distances between the two objects and the observer nor the ratio of these distances mattered.

For 2 of the observers, an effect of reference orientation was found. For these observers, the slope values of the fits were very small for two orientations $\left(22^{\circ}\right.$ and $\left.157^{\circ}\right)$ and a bit larger for the other two orientations $\left(67^{\circ}\right.$ and $\left.112^{\circ}\right)$. In other words, for these observers there was an interaction

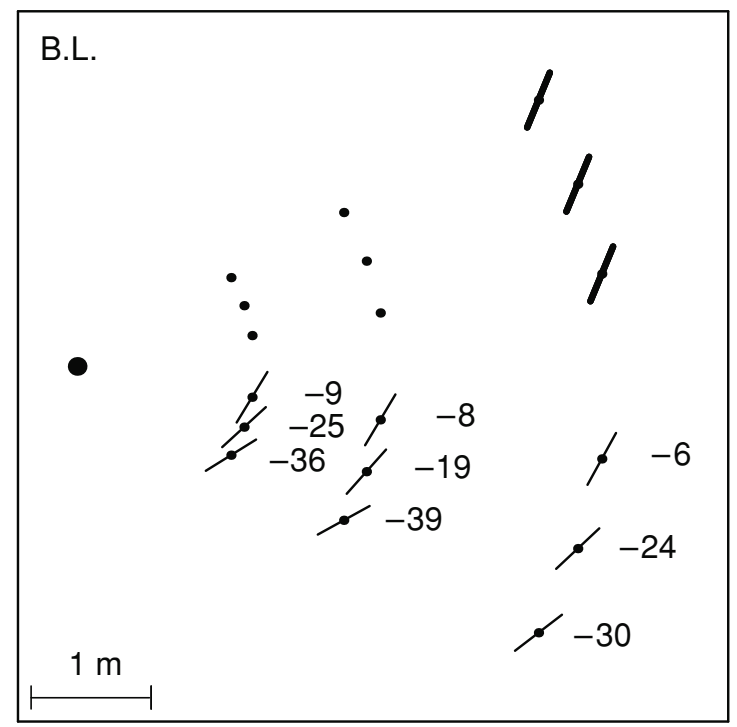

Figure 6. Schematic view of the experimental room with settings for the parallelity task. The thick lines represent the orientations of the reference rods (distance $=4.5 \mathrm{~m}$, reference orientation $=67^{\circ}$ ). The thin lines represent the means of three settings by Observer B.L. The numbers indicate deviations from the veridical setting, in degrees. 


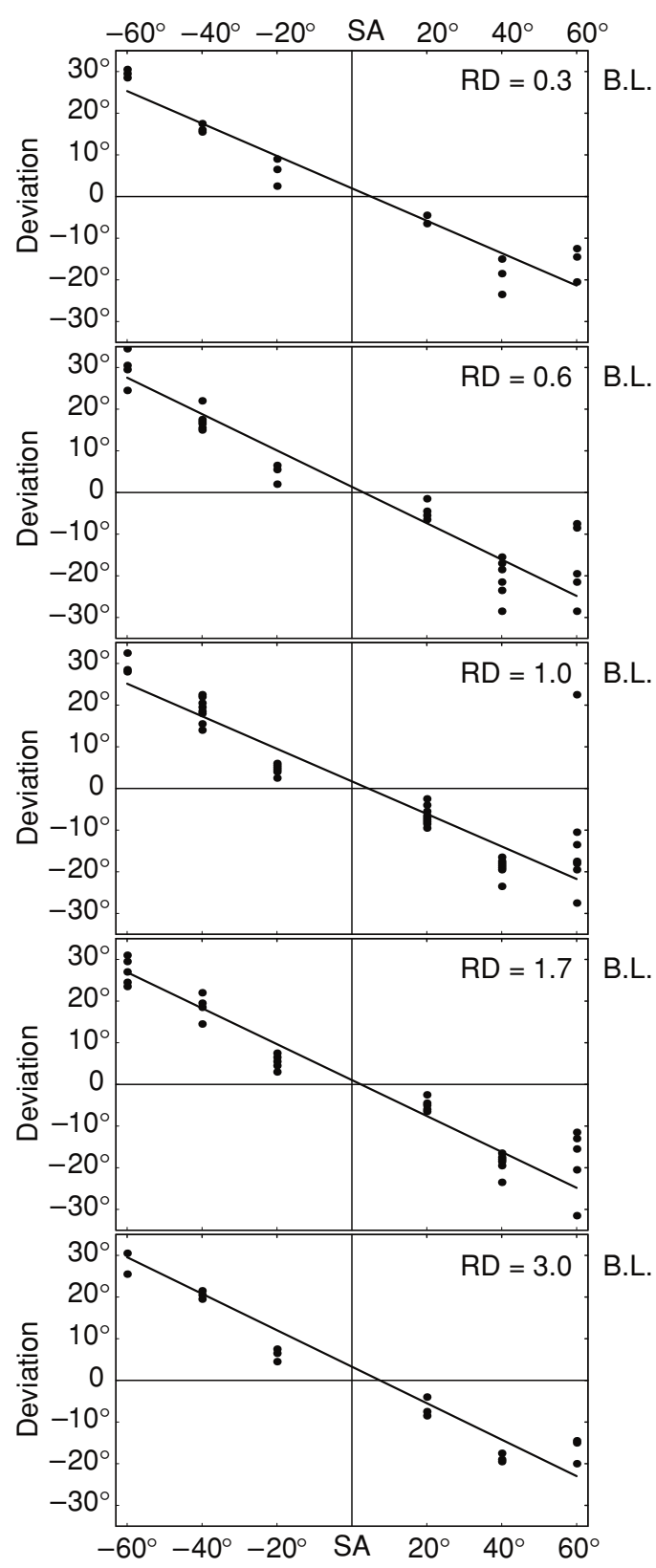

Figure 7. In each graph, the deviations from veridical settings for the parallelity task are shown as a function of separation angle (SA) for each relative distance (RD). These are the data for Observer B.L. and a reference orientation of $67^{\circ}$. A line is fitted through each set of data points.

of reference orientation with separation angle in which the size of the effect of the separation angle was dependent on the orientation of the reference rod. The difference between the observers might be due to the different kinds of information that they abstracted from the scene in order to do the task.

We compared our data with those of Cuijpers et al. (2000b). In their experiments, a linear effect of separation angle was found without any effect of relative distance.
Observers in those experiments differed greatly with regard to their dependence on reference orientation. However, in those experiments the orientations of the reference rods were different $\left(0^{\circ}, 30^{\circ}, 60^{\circ}, 90^{\circ}, 120^{\circ}\right.$, and $\left.150^{\circ}\right)$. For most of the observers, Cuijpers et al. (2000b) found that the slopes of the deviations for nonoblique orientations were negligible. As a possible explanation for this oblique effect, the researchers hypothesized that observers were able to derive some information about the $0^{\circ}$ and $90^{\circ}$ orientations from the walls of the room or the cabin in which they were seated, although an attempt had been made to conceal this information. Cuijpers and his colleagues later examined this hypothesis further by varying the orientations of the observers, the cabin in which the observers were seated, and the stimuli with respect to the walls. They found that some observers were dependent on the orientation of the walls, some were more dependent on the orientation of the cabin, and a third group was somewhere in between (Cuijpers et al., 2001). In addition to the overall oblique effect, they also found differences between the oblique orientations for some observers. For those observers, the deviations are such that for some oblique trials the resulting setting would be nonoblique. Since the perception of these nonoblique orientations is perceived as veridical, this is a conflicting situation. As a consequence, the deviations for these oblique trials for these observers are smaller than might be expected. According to this line of thinking, one would not expect to find such good fits for our data as are shown in Figure 7, because for all reference orientations one would have found smaller deviations for either the positive or the negative separation angles than for the other (depending on the orientation). This difference could be due to the multiple sources of information about the orientation of the rod in our setup. For example, an observer who is constantly looking at both the rod and the yellow disc perpendicular to it will have a different pattern of deviations than will an observer who looks primarily at the rod.

Thus, with minor exceptions, Cuijpers et al.'s (2000b) results have the same size and follow the same pattern as the results presented in this article. Therefore, for this task it can be concluded that additional context did not make observers' settings more veridical.

\section{EXPERIMENT 3 Collinearity Task}

\section{Method}

For the collinearity task, the same two rods were used as in the parallelity task. The observers used two remote controls to rotate the two rods, and their task was to align the two rods to point toward each another. The observers were instructed to rotate the rods so that they were in one line, and they were shown a picture of two collinear lines. The number of trials for this task was $81(27 \times 3$ repetitions $)$, which took the observers about $2 \mathrm{~h}$ to perform.

\section{Results}

In the collinearity task, systematic deviations were found for all observers. The size of the deviations was observer dependent. Figure 9 gives an example of settings 


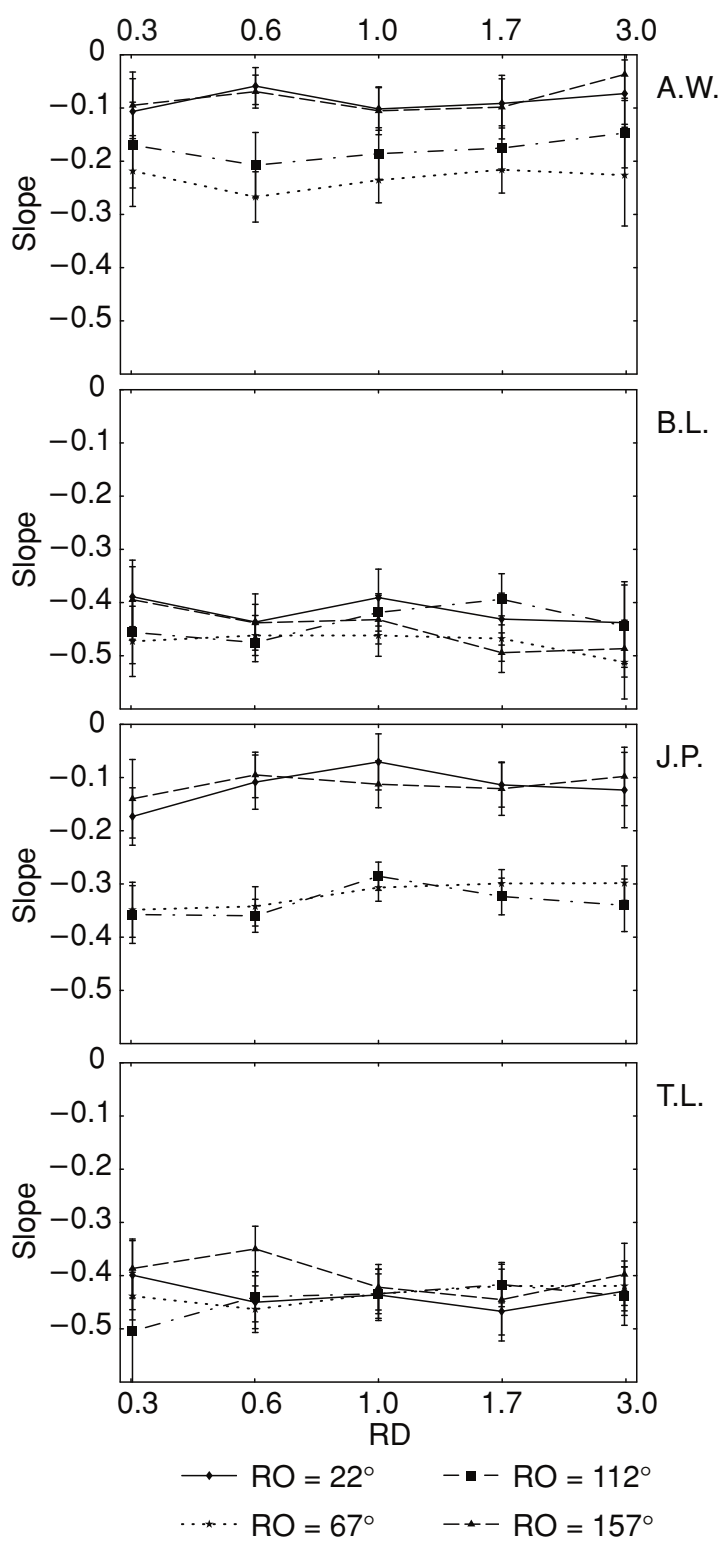

Figure 8. In each graph, the slopes of the lines for deviations from veridical settings, as given in Figure 7 for Observer B.L., are plotted as a function of relative distance (RD) for all observers. The data for the different reference orientations (RO) are given on separate lines.

for Observer A.W. The means of three repetitions of the settings are shown graphically and numerically (deviations from veridical settings in degrees) for two combinations of positions on the floor. The dotted lines are the veridical orientations of the rods.

We performed two kinds of analysis for this task. First, we looked separately at the two rods that were placed in the same trial. Second, we looked at the combination of the settings of the two rods.

Our first analysis was the same as the analysis we did for the other two tasks. We looked at the two rods sepa- rately and dealt with them in the same way as we dealt with the pointers in the pointing task. For each measurement, one rod was the test rod, and we took the center of the other rod as the target. Again, we looked at the effect of the separation angle and the relative distance on the size of the deviations and plotted the deviations against the separation angle for every relative distance (see Figure 10 for the data for Observer A.W.). A line was then fitted through these data using a least squares method. As can be seen in Figure 10, for relative distances of 0.3 and 0.6 the slope is positive, and for relative distances of 1.7 and 3.0 the slope is negative. For the relative distance of 1.0, the slope for this observer is zero. Table 3 shows the slopes of the different fits for all observers and relative distances. The asterisks indicate fits that deviate significantly from zero, which included most fits, with some exceptions. In Figure 11, slopes are plotted against the relative distances on a logarithmic scale, with the data for different observers plotted on separate lines. As can be seen in this figure, when the relative distance is smaller than 1.0, the slopes tend to be positive. This means that when the rod is closer to the observer than is the target (in this case, the middle of the other rod), the observer tends to overshoot. On the other hand, when the target is closer to the observer than is the rod, the observer tends to undershoot. The slope values are larger for the relative distances of 0.6 and 1.7 than for 0.3 and 3.0, respectively, which is the same pattern we found for the pointing task. For 2 of the observers, the slopes are zero at a relative distance of 1.0 , and for the other 2 , the slopes are negative at this relative distance.

We will discuss three different hypothetical situations that can occur if the two rods are viewed together. The first is a veridical setting of rods. The second is that the two rods are placed with deviations of opposite signs, so that both either overshoot or undershoot. The third possibility is that the rods are placed with deviations of a corresponding sign, so that one of the rods overshoots and the other undershoots. In the first situation, both the sum of and the difference between the deviations of the two rods will be zero. If the rods are placed with deviations of the same size but different signs, the sum of the deviations will be zero; this is a special case of the second possibility. If the two rods are placed parallel - that is, with deviations of the same size (not equal to zero) and sign - the difference between the deviations will be zero, but the sum will not be zero. This is a special case of the third possibility.

Table 2

Slopes of the Linear Fits Through the Data Points as a Function of Relative Distance for All Observers in the Parallelity Task

\begin{tabular}{ccc} 
& \multicolumn{2}{c}{ Angle } \\
\cline { 2 - 3 } Observer & $22^{\circ} / 157^{\circ}$ & $67^{\circ} / 112^{\circ}$ \\
\hline A.W. & -0.08 & -0.20 \\
B.L. & -0.43 & -0.46 \\
J.P. & -0.11 & -0.32 \\
T.L. & -0.42 & -0.44 \\
\hline
\end{tabular}

Note-The slopes are the means of the slopes found for all relative distances and two reference orientations. 


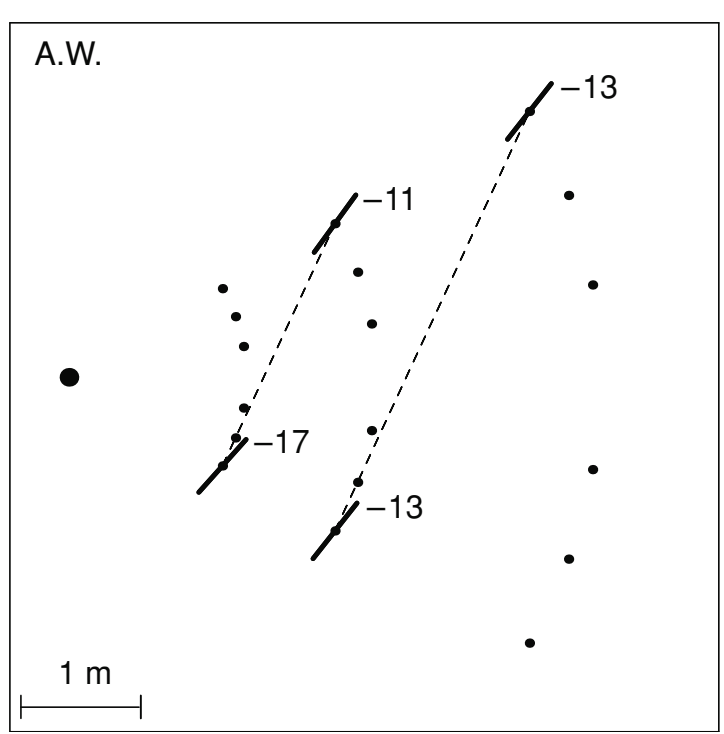

Figure 9. Schematic view of the experimental room with settings for the collinearity task. The dashed lines represent the veridical settings for conditions in which the separation angle was $-60^{\circ}$ and the relative distance was $0.6 / 1.7$. The solid lines represent the means of the settings for three trials by Observer A.W. The numbers indicate the deviations from the veridical settings, in degrees.

In Figure 12, we have plotted the sum of the deviations of corresponding rods as a function of the relative distance (on a logarithmic scale). Separate plots show the data for the 4 different observers; each point represents the mean of three repetitions, and for some relative distances a point represents a couple of combinations of points that have the same relative distance. Standard deviations are given via error bars. What can be seen in the graphs is that the sign of the sum changes when the relative distance changes from smaller to larger than 1.0. At a relative distance of 1.0, the sum is zero for all observers. The sum is larger for relative distances of 0.6 and 1.7 than for relative distances of 0.3 and 3.0, respectively, and for the smallest separation angle $\left(20^{\circ}\right)$ the relative distance had only a minor effect.

The difference between the deviations of the two bars is plotted in Figure 13 against the relative distance. Again, the separate graphs represent the different observers, relative distance is given on a logarithmic scale, and the error bars represent standard deviations. Overall, the differences between the deviations are smaller than the sums and are not dependent on relative distance. For Observers A.W. and T.L., at a relative distance of 1.0 the difference is zero. Since the sum is also zero, this means that the settings for this relative distance were veridical. For the other 2 observers, the difference is not equal to zero at a relative distance of 1.0. Since the sums for these observers are zero at that distance, this means that the data were symmetrical. There was only a minor effect of separation angle on the difference between the deviations.

\section{Discussion}

First, we will discuss the analysis of the two rods taken separately. We performed this analysis mainly to compare with the results of the exocentric pointing task. The same pattern was found for both tasks: As with the pointing task, the data of the collinearity task revealed a linear effect of the separation angle (see Figure 10). There was also an effect of relative distance, in that there was an undershoot when the relative distance was smaller than 1.0 and an overshoot when the relative distance was larger than 1.0 (see Figure 11). Larger deviations were found for relative distances of 0.6 and 1.7 than for relative distances of 0.3 and 3.0, with the possible explanation that, as in the pointing task, the more the relative distance deviates from 1.0, the more egocentric the exocentric task becomes.

For 2 of our observers, A.W. and T.L., we found (close to) veridical settings for a relative distance of 1.0. For the other 2, we found settings with an overshoot. This pattern is the same as the one we found for the pointing task, in which the same 2 observers produced veridical settings.

When we analyzed the two rods together, we found that the sum of the deviations was dependent both on relative distance and separation angle. This finding is comparable to that of Cuijpers et al. (2002). On the other hand, the difference was fairly constant over different relative distances (with the exception of 2 observers who had a difference of zero when the relative distance was 1.0) and depended slightly on the separation angle. Cuijpers et al. (2002) also found a constant difference, without even the slight dependency on separation angle. Because (at least in geometry) collinearity is a special case of parallelity, one would expect the differences found between the settings of the two bars in the collinearity task to be of the same size as the deviations found in the parallelity task. This was not the case in the present experiments, however; overall, the differences found for the collinearity task were smaller than the deviations found for the parallelity task, and the pattern of deviations was also qualitatively different. The same discrepancy between the two tasks was found by Cuijpers et al. (2002), showing that the geometrically similar parallelity and collinearity tasks are fundamentally different for the human observer.

\section{GENERAL DISCUSSION}

As can be seen directly from the graphs (see Figures 5 and 11), the results of the exocentric pointing task are very similar to the results of the collinearity task. The same size and pattern of deviations was found for both. Even the distinction between two groups of observers, when one looks at the deviations found for a relative distance of 1.0, is the same. At this relative distance, the same observers (A.W. and T.L.) have veridical settings for both tasks. In contrast, close to veridical settings for this condition were not found so clearly by Cuijpers et al. (2000a, 2002). An explanation for this difference could be as follows: When both objects were at the same distance from the observer, 


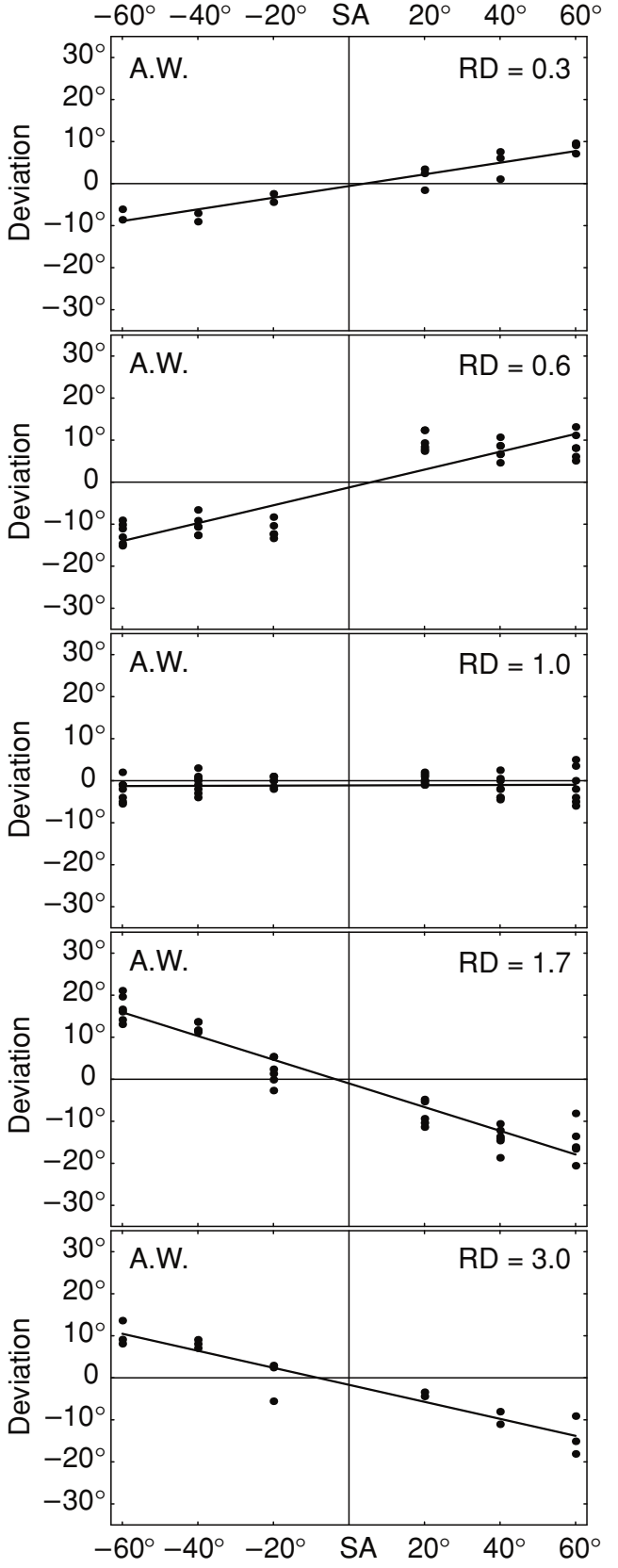

Figure 10. In each graph, the deviations from veridical settings for the collinearity task are shown as a function of separation angle (SA) for each relative distance (RD). These are the data for Observer A.W. A line is fitted through each set of data points.

the veridical pointing direction was parallel to the back wall, so for this condition, direct information from the context was available for the observer to do the setting.

If we compare these data with those from the parallelity task (see Figure 8), we see a very different pattern. One of the differences is the size of the deviations, which were larger for the parallelity task than for the other two tasks. In the parallelity task, the orientations of the reference rod and the test rod can be misjudged, but in the pointing task only the orientation of the test rod (the pointer) can be misjudged, because there is no reference rod. The collinearity task can be split into two parts - pointing from one rod to the other, and vice versa - so the orientation of one rod is not so dependent on the orientation of the other rod as it is in the parallelity task. According to this reasoning, one would expect the deviations in the parallelity task to be twice the size of the deviations in the other two tasks, which was exactly what we found in our experiments. A second difference is the effect of relative distance: Unlike with the other tasks, for the parallelity task no effect of relative distance was found. This distinction was also found by Cuijpers et al. (2000a, 2000b, 2002).

These quantitative and qualitative distinctions between the parallelity task and the collinearity task seems quite strange when one considers that in geometry, collinearity is a special case of parallelity. However, when one compares the two tasks, one can distinguish the ways in which the tasks are performed. For the parallelity task, the observer does not have to identify the exact positions of the two objects; only their orientations are important. On the other hand, for the collinearity task, matching the orientations is not sufficient; an essential part of the task is to take into account the spatial relationship between the two objects. The collinearity task resembles a pointing task in which observers must point with one pointer to the center of the other pointer, so it is perhaps not surprising to find a pattern and size of deviations that are comparable to those found for the pointing task.

This comparison of the three tasks indicates that the concept of a single visual space is problematic. Apparently, "visual space" is deformed differently depending on the information in the environment necessary to do a certain task. For instance, one can describe the settings of the parallelity task geometrically by means of only the separation angle and the reference orientation; the distance information is irrelevant for this task. This is totally different from the dependence on relative distance in the exocentric pointing and collinearity tasks. Thus, it is to be expected that human observers would perform differently in this task in comparison with the other tasks. Furthermore, one would expect to find more veridical settings for the pointing and collinearity tasks in our data than in Cuijpers et al.'s, since our observers had increased information about depth from monocular cues such as linear perspective and texture segregation. In contrast, perfor-

Table 3

Slopes of the Linear Fits Through the Data Points as a Function of Relative Distance for All Observers in the Collinearity Task

\begin{tabular}{lccccc}
\hline & \multicolumn{5}{c}{ Relative Distance } \\
\cline { 2 - 6 } Observer & 0.3 & 0.6 & 1.0 & 1.7 & 3.0 \\
\hline A.W. & $0.14^{*}$ & $0.21^{*}$ & 0 & $-0.28^{*}$ & $-0.20^{*}$ \\
B.L. & $-0.09^{*}$ & $0.26^{*}$ & $-0.10^{*}$ & $-0.28^{*}$ & $-0.21^{*}$ \\
J.P. & 0.02 & $0.09^{*}$ & $-0.09^{*}$ & $-0.18^{*}$ & $-0.09^{*}$ \\
T.L. & $-0.11^{*}$ & 0.01 & 0 & $-0.17^{*}$ & -0.06 \\
\hline
\end{tabular}

Note-The data for A.W. are illustrated in Figure 10. ${ }^{*}$ The slope deviates significantly from $0(\alpha=.05)$. 


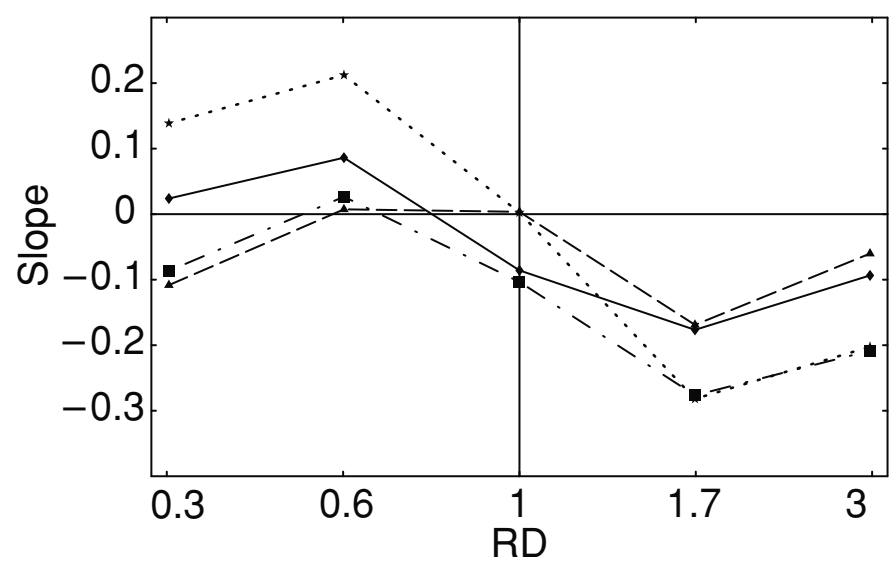

$\ldots \ldots$ A.W. -- B.L. $\multimap$ J.P. -- T.L.

Figure 11. The slopes of the lines for deviations from veridical settings, as given in Figure 10 for Observer A.W., are plotted as a function of relative distance (RD) for all observers. The data for the different observers are given on separate lines.

mance for the parallelity task is less dependent on such distance information.

In the parallelity task, a different degree of dependence on reference orientation was found for our observers. Cuijpers et al. (2001) discussed a difference between observers in dependence on references like the walls or the cabin the observers were seated in, but this difference mainly reflected a distinction between oblique and nonoblique orientations, so this hypothesis cannot explain our finding of differences between various oblique angles. Cuijpers et al. (2000b) noted a small difference between observers for oblique settings, but their explanation also does not fit our data. An alternative explanation might relate to the degree of change in the view of an object when an object is rotated a small amount. For example, when an observer looks only at a rod that is perpendicular to the line of sight, a rotation of $5^{\circ}$ does not change the image of the rod on the retina as much as it would change the

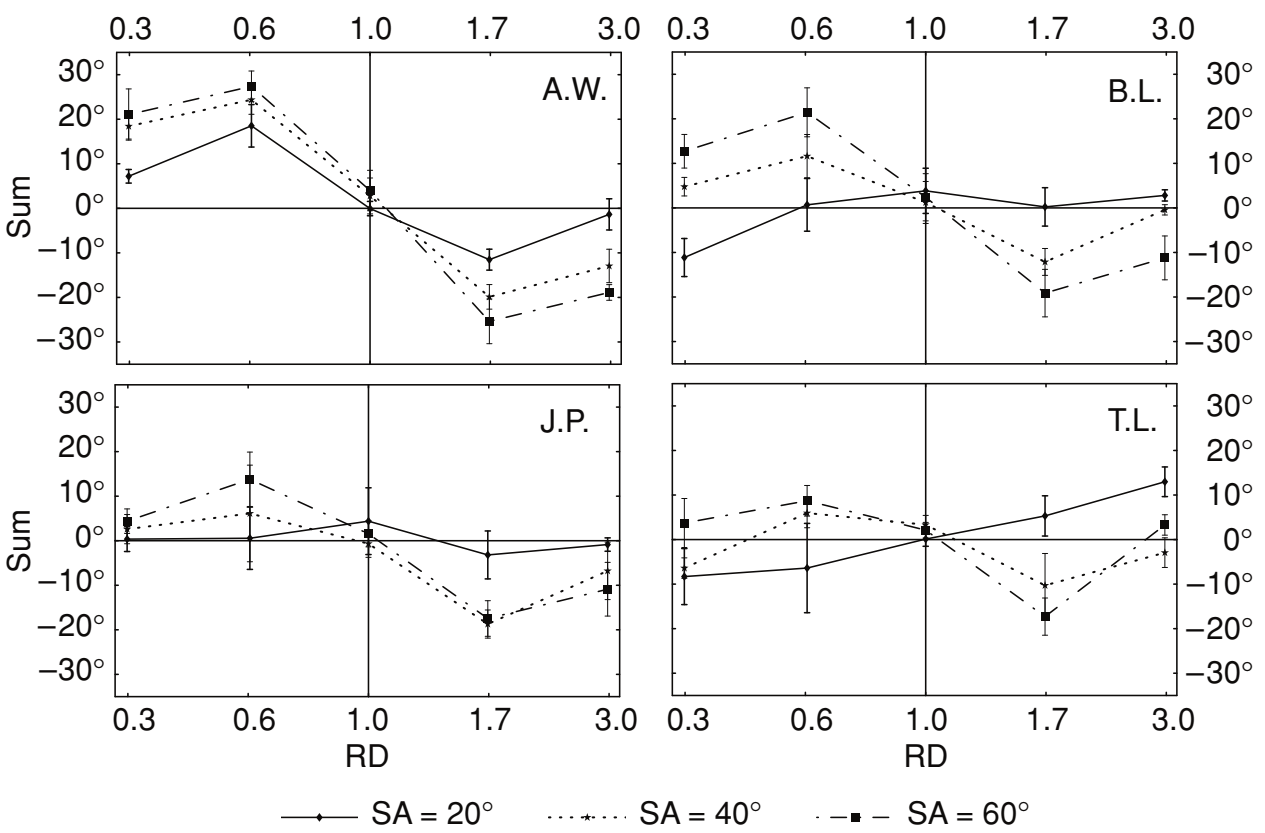

Figure 12. In each graph, the sums of deviations are plotted against relative distance (RD) for 1 observer. Standard deviations are given via error bars. The different lines represent different separation angles (SA). 

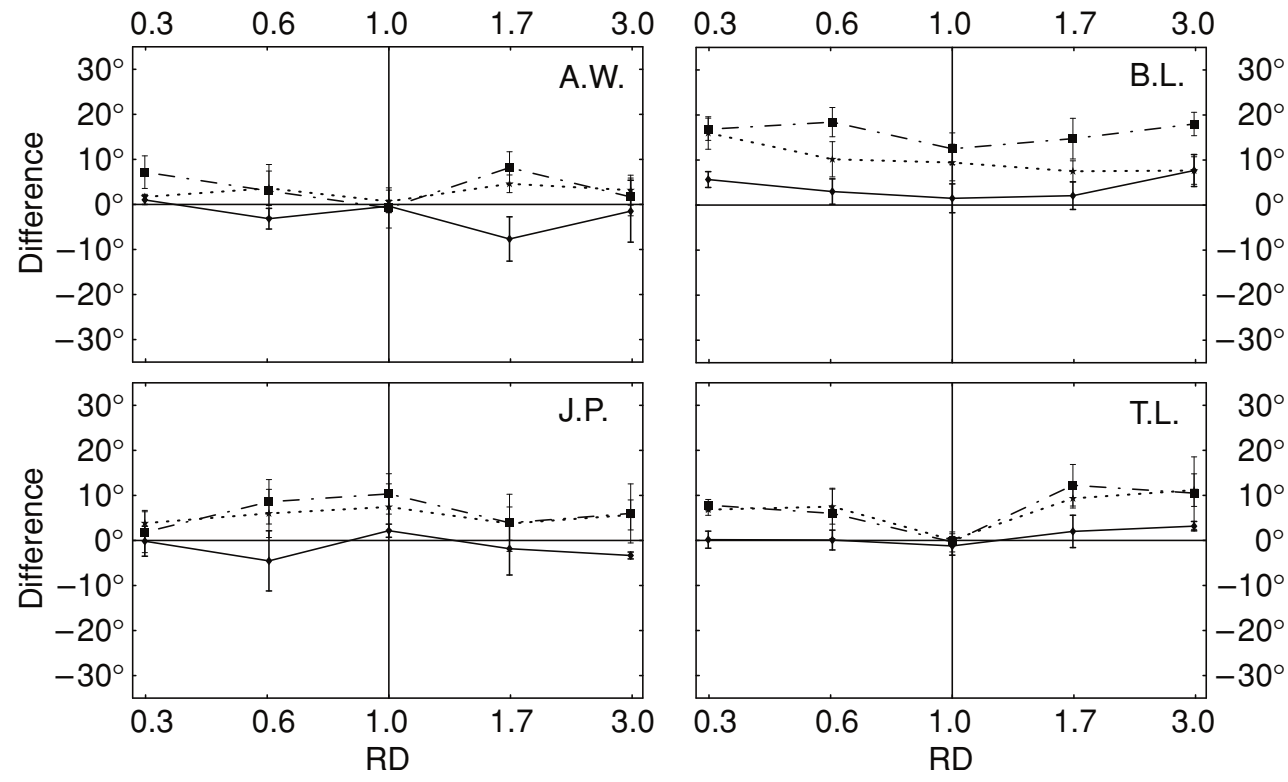

$$
\because \mathrm{SA}=20^{\circ} \quad \cdots * \cdots \mathrm{SA}=40^{\circ} \quad-\quad-\mathrm{SA}=60^{\circ}
$$

Figure 13. In each graph, the differences between deviations are plotted against relative distance (RD) for 1 observer. Standard deviations are given via error bars. The different lines represent different separation angles (SA).

image of a rod that was collinear with the line of sight. On the other hand, if an observer looks both at the rod and the disc around it, the attended image on the retina will always have a rather large change. Thus, this difference in the amount of change depends upon the sources of information people use when performing a task.

For the pointing and collinearity tasks, the veridical setting of the pointers can be described using the following formula:

$$
\tan \beta=\frac{\sin \alpha}{\frac{r_{1}}{r_{2}}-\cos \alpha},
$$

where $\beta$ is the angle between the line connecting the pointer and the target and the line connecting the pointer and the observer (Koenderink, van Doorn, \& Lappin, 2003). To use this formula for the collinearity task, we define one rod as the pointer, and the middle of the other rod can be defined as the target. The variable $\alpha$ represents the separation angle between the two objects, and $r_{1}$ and $r_{2}$ represent the distances from the observer to the pointer and the target, respectively. Traditionally, the focus has been on the perceived distances and how they are derived from the physical distances $r_{1}$ and $r_{2}$. Different models have been fitted to different data sets. For example, Wagner (1985) compared four models using data acquired under full-cue conditions. Two of these were Riemannian models, one spherical and the other hyperbolic. Neither of these models fit his data: The spherical model produced such strange fits that it was rejected, and the hyperbolic model did not produce good fits either, which was ex- plained by noting that the model was made for reducedcue conditions. Another model Wagner described was an affine contraction model, which describes an affine transformation in depth (only in the direction straight ahead) using Cartesian metrics. Because humans are not thought to depend on Cartesian coordinates in dealing with depth, this model was refined into the vector compression model, which uses polar coordinates and multiplies the physical distance by a constant. This model fit Wagner's data very well. In Equation 2, however, the physical distance constant would cancel itself out in the ratio that represents the relative distance. Thus, since multiplication of the distances by a constant has no effect on the pointing direction, the vector compression model cannot explain our results. We also looked at Gilinsky's formula for perceived distance (Gilinsky, 1951; see Equation 1), replacing $r_{1}$ and $r_{2}$ from Equation 2 with $P\left(r_{1}\right)$ and $P\left(r_{2}\right)$. This revised equation did not fit our data well, but that is not surprising, since Gilinsky formulated her theory on the basis of data obtained with larger distances and described visual space as compressed. Such compression of visual space does not suit our data obtained with smaller distances. In fact, for 2 of our observers we found a (bad) fit with a negative constant, which is nonsense in the Gilinsky formula, since negative values of $c$ would indicate an expansion, not a compression, of space. Therefore, for the tasks in which spatial information was most important, the settings should be described by an expanding distance function (like a power law) rather than by a compressing function. This difference, possibly due to the varying distances used, is consistent with ideas proposed by Battro 
et al. (1976) and Koenderink et al. (2000) that geometry varies with the distance form observer to objects.

The data described above are quite similar to those found by Cuijpers et al. (2000a, 2000b, 2002). This result is not what we expected, since our experiments were conducted in a very different environment. In our experiments, the walls, ceiling, floor, radiators, windows, doors, and so on were all visible, in contrast with the environment used by Cuijpers et al. Despite the extra information provided by linear perspective, texture segregation, size constancy, and so forth, our observers nonetheless showed a magnitude and pattern of deviations comparable to those found by Cuijpers et al. This result could be explained in the following way: Perhaps the structure that was provided to the observers was not rich enough, and a richer structural context would make a difference. Wagner (1985) talked about the quantity and the quality of depth information and concluded that if both were maximal, visual space should be Euclidean. If one reduces both the quantity and quality of perceptual information, deformation of visual space will increase as well. A next step in this research should thus be to elaborate the context provided to observers and see whether the deformation of visual space will decrease. For example, we could put textures on the walls and floors or place extra objects in the room.

In summary, one cannot speak of a single visual space, since the structure is dependent on the observer's task and the distance between the objects and the observer. The structure of visual space for two tasks that require spatial information for the objects (the exocentric pointing task and the collinearity task) and a distance of 1.5 to $4.5 \mathrm{~m}$ between observers and objects can be described by an expanding distance function, such as a power law. For the parallelity task, a distance function is useless, since the information about the exact positions of the objects is not necessary to do the task. Another conclusion that can be drawn from these data is that the settings of the observers in this environment full of monocular depth cues were similar to the settings found for data obtained in a much poorer environment. Thus, the structure in this richer environment was not used effectively by the observers.

\section{REFERENCES}

Battro, A. M., di Pierro Netto, S., \& Rozestraten, R. J. A. (1976). Riemannian geometries of variable curvature in visual space: Visual alleys, horopters, and triangles in big open fields. Perception, 5, 923.

BlanK, A. A. (1961). Curvature of binocular visual space: An experiment. Journal of the Optical Society of America, 51, 335-339.

Cuijpers, R. H., Kappers, A. M. L., \& Koenderink, J. J. (2000a). Investigation of visual space using an exocentric pointing task. Perception \& Psychophysics, 62, 1556-1571.

Cuijpers, R. H., Kappers, A. M. L., \& Koenderink, J. J. (2000b). Large systematic deviations in visual parallelity. Perception, 29, 1467-1482.

Cuijpers, R. H., Kappers, A. M. L., \& Koenderink, J. J. (2001). On the role of external reference frames on visual judgements of parallelity. Acta Psychologica, 108, 283-302.

Cuijpers, R. H., Kappers, A. M. L., \& Koenderink, J. J. (2002). Visual perception of collinearity. Perception \& Psychophysics, 64, 392-404.

Gibson, J. J. (1950). The perception of the visual world. Cambridge: Riverside Press.

GiLINSKY, A. S. (1951). Perceived size and distance in visual space. Psychological Review, 58, 460-482.

Helmholtz, H. L. F. (1962). Helmholtz's treatise on physiological optics (J. P. C. Southall, Trans.). New York: Dover. (Original work published 1866)

INDOw, T. (1991). A critical review of Luneburg's model with regard to global structure of visual space. Psychological Review, 98, 430-453.

IndOW, T., \& Watanabe, T. (1984). Parallel-alleys and distance-alleys on horopter plane in the dark. Perception, 13, 165-182.

Indow, T., \& Watanabe, T. (1988). Alleys on an extensive apparent frontoparallel plane: A second experiment. Perception, 17, 647-666.

Koenderink, J. J., van Doorn, A. J., Kappers, A. M. L., \& LapPin, J. S. (2002). Large-scale visual frontoparallels under full-cue conditions. Perception, 31, 1467-1475.

Koenderink, J. J., van Doorn, A. J., \& Lappin, J. S. (2000). Direct measurement of the curvature of visual space. Perception, 29, 69-79.

Koenderink, J. J., van Doorn, A. J., \& Lappin, J. S. (2003). Exocentric pointing to opposite targets. Acta Psychologica, 112, 71-87.

LuneBURG, R. K. (1950). The metric of binocular visual space. Journal of the Optical Society of America, 40, 627-640.

Wagner, M. (1985). The metric of visual space. Perception \& Psychophysics, 38, 483-495.

ZAJACZKOWSKA, A. (1956). Experimental determination of Luneburg's constants $\sigma$ and K. Quarterly Journal of Experimental Psychology, 8, 66-78.

(Manuscript received November 26, 2003; revision accepted for publication December 15, 2004.) 